\title{
Adomian's Decomposition Method to Squeezing Flow and Heat Transfer between two Parallel Disks with Velocity Slip and Temperature Jump
}

\author{
Muhammad Usman, Aqsa Nazir, Zertaisha Naheed, Syed Tauseef Mohyud-Din* \\ Department of Mathematics, HITEC University, Taxila Cantt Pakistan \\ *Corresponding Author: syedtauseefs@hotmail.com
}

Copyright (C) 2013 Horizon Research Publishing All rights reserved.

\begin{abstract}
In this paper, we are apply Adomian's Decomposition Method to find appropriate solutions of to squeezing flow and heat transfer between two parallel disks with velocity slip, temperature jump which are of utmost importance in applied and engineering sciences. A concrete relation between the basic ideas of the proposed technique and the existing literature is also presented. Numerical results coupled with graphical representation explicitly reveal the complete reliability of the proposed algorithm.
\end{abstract}

Keywords MHD Squeezing Flow, Newtonian Fluid, Velocity Slip, Temperature Jump, Adomian's Decomposition Method, Adomian's Polynomials, Numerical Solution

\section{Introduction}

In recent years, squeezing flow of Newtonian and non-Newtonian fluids has attracted considerable attention of many researchers because of its widespread applications in different engineering disciplines. Such flows are induced by two approaching parallel surfaces in relative motion. Stefan [1] was the first to initiate pioneering work on the squeezing flow by invoking lubrication approach. Kuzma [2] studied the effects of fluid inertia in squeeze films between two circular glass plates. Magneto hydrodynamic squeeze film was considered by Hamza [3]. Recently, Domairry and Aziz [4] employed He's homotopy perturbation method to consider the suction and injection effects on the flow of electrically conducting viscous fluid squeezed between two parallel disks. Hayat et al. $[5,6]$ extended the work presented in [4] to analyze the squeezing flow of non-Newtonian fluids by taking second grade and micropolar models. While Qayyum et al. [7] investigated the unsteady squeezing flow of viscoelastic Jeffery fluid between parallel disks and discussed the porosity and squeezing effects on the velocity profile. Besides, some authors studied the heat transfer characteristics of viscous fluid in a squeezing flow between two parallel surfaces. Khaled and Vafai [8] analyzed the hydromagnetic effects on flow and heat transfer over a horizontal surface placed in externally squeezed free stream. They found that local Nusselt number and local wall shear stress are both increasing functions of magnetic parameter. Squeezing flow and heat transfer over a porous surface was investigated by Mahmood et al. [9]. Duwairi et al. [10] examined the effects of heat transfer while Mustafa et al. [11] considered the combined effects of heat and mass transfer in a viscous fluid squeezed between two parallel plates. In [12], the authors obtained the radial solution to the problem of steady squeezing flow and heat transfer between parallel disks. More recently, Bahadir and Abasov [13] employed the finite difference approach to obtain numerical solution of hydromagnetic steady flow and heat transfer in a viscous fluid squeezed between two parallel disks. One of the core concepts of fluid mechanics is the no slip boundary conditions, i.e. the assumption that when a liquid flows over a solid surface, the liquid molecules adjacent to the solid are stationary relative to the solid [14]. This no slip boundary condition has been utilized in modelling several flow problems of viscous and viscoelastic fluids. The condition was first questioned by Navier [15], who suggested the general boundary condition which shows the fluid slip at solid-liquid interface. According to him the amount of liquid slip at the boundary is proportional to the shear stress at that boundary. The constant of proportionality is known as velocity slip parameter which has the dimension of length. The slip boundary condition has direct implications for nanofluidics. Some important positive effects of the slip in nanofluidics are the reduction of friction, energy conservation and mimicking biological water channels [16]. Keeping in mind these facts many researchers are now investigating the effects of velocity slip, temperature jump and other newly developed issues on flow and heat transfer characteristics of viscous fluids in different configurations. A number of significant studies have been undertaken in this regard, including peristaltic transport of a Newtonian fluid in 
an asymmetric channel [17], viscous flow due to a stretching sheet with surface slip and suction [18], boundary layer stagnation-point flow and heat transfer towards a shrinking sheet [19], MHD liquid flow and heat transfer over non-linear permeable stretching surface with chemical reaction [20], Three dimensional rotating flow and heat transfer between two porous wall [21], and MHD flow and heat transfer over a porous shrinking surface [22]. The study reported here extends the work of Domairry and Aziz [4] by considering the velocity slip and temperature jump at the surfaces of lower and upper disks. Our principle goal is to study the effects of slip parameters on the velocity and temperature profiles and on the related physical quantities like skin friction coefficient and the Nusselt number. Adomian's decomposition method [23-27] is used to obtain the numerical solution of the nonlinear flow problem. Numerical solution is also computed and comparison is made between the two sets of results.

\section{Basic Equations and Mathematical Formulation}

Consider the axisymmetric flow of an incompressible electrically conducting viscous fluid squeezed between two parallel disks separated by a variable distance $h(t)=$ $H \sqrt{1-\alpha t}$. Where $\alpha$ is a characteristic parameter having dimensions that of time inverse. The upper disk at $z=h(t)$ is approaching the lower stationary disk at $z=0$ with a velocity $v(t)=\frac{d h}{d t}$. We consider the velocity profile in the form $(u, 0, w)$, where $u$ and $w$ are radial and axial velocities along $r$ and $z$ axes, respectively. A uniform magnetic field proportional to $\frac{B_{0}}{\sqrt{1-\alpha t}}$ is applied along the axial component and electric field is taken zero. Under the stated conditions the continuity, momentum and energy equations can be written as:

$$
\begin{aligned}
& \frac{\partial u}{\partial r}+\frac{u}{r}+\frac{\partial w}{\partial z}=0, \\
& \rho\left(\frac{\partial u}{\partial t}+u \frac{\partial u}{\partial r}+w \frac{\partial u}{\partial z}\right)=-\frac{\partial p}{\partial r}+\mu\left(\frac{\partial^{2} u}{\partial r^{2}}+\frac{1}{r} \frac{\partial u}{\partial r}+\frac{\partial^{2} u}{\partial z^{2}}-\frac{u}{r^{2}}\right)-\frac{\sigma B_{0}^{2}}{1-\alpha t} u \text {, } \\
& \rho\left(\frac{\partial w}{\partial t}+u \frac{\partial w}{\partial r}+w \frac{\partial w}{\partial z}\right)=-\frac{\partial p}{\partial z}+\mu\left(\frac{\partial^{2} w}{\partial r^{2}}+\frac{1}{r} \frac{\partial w}{\partial r}+\frac{\partial^{2} w}{\partial z^{2}}\right), \\
& \rho C_{p}\left(\frac{\partial T}{\partial t}+u \frac{\partial T}{\partial r}+w \frac{\partial T}{\partial z}\right)=k\left(\frac{\partial^{2} T}{\partial r^{2}}+\frac{1}{r} \frac{\partial T}{\partial r}+\frac{\partial^{2} T}{\partial z^{2}}\right) \\
& +2 \mu\left[\left(\frac{\partial u}{\partial r}\right)^{2}+\left(\frac{u}{r}\right)^{2}+\left(\frac{\partial w}{\partial z}\right)^{2}+\frac{1}{2}\left(\frac{\partial u}{\partial z}+\frac{\partial w}{\partial r}\right)^{2}\right],
\end{aligned}
$$

subject to the boundary conditions

$$
\begin{gathered}
u=\beta_{1} \frac{\partial u}{\partial z}, w=0, \quad T=\gamma_{1} \frac{\partial T}{\partial z}+T_{0}, \quad \text { at } z=0, \\
u=-\beta_{1} \frac{\partial u}{\partial z}, \quad w=\frac{d h(t)}{d t}, \quad T=-\gamma_{1} \frac{\partial T}{\partial z}+T_{1}, \quad \text { at } z=h(t),
\end{gathered}
$$

where $\rho$ is fluid density, $\mu$ is dynamic viscosity, $p$ is the pressure, $\sigma$ is electrical conductivity, $C_{p}$ is the specific heat at constant pressure, $k$ is the thermal conductivity, $\beta_{1}$ and $\gamma_{1}$ are the slip parameters with respect to velocity and temperature. Note that $T, T_{0}$ and $T_{1}\left(T_{1}>T_{0}\right)$ are the temperature of the fluid, constant temperatures of the lower and upper disks, respectively.

Introducing the dimensionless quantities:

$$
\begin{aligned}
u=\frac{\alpha r}{2(1-\alpha t)} f^{\prime}(\eta), w= & -\frac{\alpha H}{\sqrt{1-\alpha t}} f(\eta) \\
& , T=T_{0}+\left(T_{1}-T_{0}\right) \theta(\eta), \eta=\frac{z}{H \sqrt{1-\alpha t}} .
\end{aligned}
$$

Substituting $u$ and $w$ into Eqs. (2-3) and eliminating the pressure gradient, we obtain the following dimensionless differential equation:

$$
\begin{aligned}
f^{\prime \prime \prime \prime}(\eta)-S\left(\eta f^{\prime \prime \prime}(\eta)+3 f^{\prime \prime}(\eta)-2 f(\eta) f^{\prime \prime \prime}(\eta)\right) & \\
& -M^{2} f^{\prime \prime}(\eta)=0 .
\end{aligned}
$$

By virtue of (6), the energy equation (4) and the boundary conditions (5) take the following form

$$
\begin{aligned}
& \theta^{\prime \prime}(\eta)+\operatorname{Pr} S\left(2 f(\eta) \theta^{\prime}(\eta)-\eta \theta^{\prime}(\eta)\right)+\operatorname{Pr} E c\left(f^{\prime \prime}(\eta)^{2}+12 \delta^{2} f^{\prime}(\eta)^{2}\right)=0 . \\
& f(0)=0, f^{\prime}(0)-\beta f^{\prime \prime}(0)=0, \quad \theta(0)-\gamma \theta^{\prime}(0)=0, \\
& f(1)=\frac{1}{2}, f^{\prime}(1)+\beta f^{\prime \prime}(1)=0, \quad \theta(1)+\gamma \theta^{\prime}(1)=1 \text {, }
\end{aligned}
$$


where the respective values of squeeze number $S$, the Hartman number $M$ the Prandtl number Pr the Eckert number $E c$, the dimensionless slip parameters $\beta$ and $\gamma$ with respect to velocity and temperature, and the dimensionless number $\delta$ are

$$
\begin{gathered}
S=\frac{\alpha H^{2}}{2 v}, M^{2}=\frac{\sigma H^{2} B_{0}^{2}}{\mu}, \operatorname{Pr}=\frac{\mu C_{p}}{k}, E c=\frac{1}{C_{p}\left(T_{1}-T_{0}\right)}\left(\frac{\alpha r}{2(1-\alpha t)}\right), \\
\beta=\frac{\beta_{1}}{H \sqrt{1-\alpha t}}, \gamma=\frac{\gamma_{1}}{H \sqrt{1-\alpha t}}, \delta=\frac{H \sqrt{1-\alpha t}}{r} .
\end{gathered}
$$

The dimensionless physical quantities like skin friction coefficient and local Nusselt number can be calculated from the following:

$$
C_{f r}=\frac{\left.\mu \frac{\partial u}{\partial z}\right|_{z=h(t)}}{\rho[v(t)]^{2}}, N u=-\frac{\left.H k \frac{\partial T}{\partial z}\right|_{z=h(t)}}{k\left(T_{1}-T_{0}\right)},
$$

which by virtue of (6) reduces to

$$
\sqrt{1-\alpha t} \frac{H^{2}}{r^{2}} R_{e r} C_{f r}=f^{\prime \prime}(1), \sqrt{1-\alpha t} N u=-\theta^{\prime}(1),
$$

where $R_{e r}=\frac{\alpha H r}{2 v}$ is the local squeeze Reynolds number.

\section{Analysis of Method}

Consider the following nonlinear differential equation

$$
L u+R u+N u=g,
$$

where $L$ is, mostly the lower order derivative which is assumed to be invertible, $R$ is other linear differential operator, non linear term and $g$ is a source term. We next apply the inverse operator $L^{-1}$ to both sides of equation (11) and using the given condition to obtain,

$$
u=f-L^{-1}(R u+N u),
$$

where the function $f$ represents the terms arising from integrating the source term $g$ and from using the given conditions that are assumed to be prescribed. As indicated before, Adomian's method defines the solution $u$ by an infinite series of components given by,

$$
u(x, y)=\sum_{n=x 0}^{\infty} u_{n}(x, y),
$$

where the components $u_{0}, u_{1}, u_{2}$...are usually recurrently determined. Substituting (13) into both sides of (12) leads to,

$$
\sum_{n=0}^{\infty} u_{n}=f-L^{-1}\left(R\left(\sum_{n=0}^{\infty} u_{n}\right)+N\left(\sum_{n=0}^{\infty} u_{n}\right)\right) \text {, }
$$

accordingly, the formal recursive relation is defined by,

$$
\begin{gathered}
u_{0}=f \\
u_{k+1=}-L^{-1}\left(R u_{k}+N u_{k}\right) .
\end{gathered}
$$

\section{Solution of Problem}

Applying ADM the equation (7) and (8) in operator form will be given as

$$
\begin{gathered}
L_{1} f=S\left(\eta f^{\prime \prime \prime}+3 f^{\prime \prime}-2 f f^{\prime \prime \prime}\right)+M^{2} f^{\prime \prime}, \\
L_{2} \theta=-\operatorname{Pr} S\left(2 f \theta^{\prime}-\eta \theta^{\prime}\right)-\operatorname{Pr} E c\left(f^{\prime \prime 2}+12 \delta^{2} f^{\prime 2}\right),
\end{gathered}
$$

where $L_{1}=\frac{d^{4}}{d \eta^{4}}$, and $L_{2}=\frac{d^{2}}{d \eta^{2}}$.

Applying $L_{1}^{-1}$ and $L_{2}^{-1}$ on both sides of above equations, we get,

$$
\begin{gathered}
f(\eta)=f(0)+\eta f^{\prime}(0)+\frac{\eta^{2}}{2} f^{\prime \prime}(0)+\frac{\eta^{3}}{3 !} f^{\prime \prime \prime}(0)+L_{1}^{-1}\left\{S\left(\eta f^{\prime \prime \prime}+3 f^{\prime \prime}-2 f f^{\prime \prime \prime}\right)+M^{2} f^{\prime \prime}\right\}, \\
\theta(\eta)=\theta(0)+\eta \theta^{\prime}(0)+L_{2}^{-1}\left\{-\operatorname{Pr} S\left(2 f \theta^{\prime}-\eta \theta^{\prime}\right)-\operatorname{PrEc}\left(f^{\prime \prime 2}+12 \delta^{2} f^{\prime 2}\right)\right\},
\end{gathered}
$$

where $L_{1}^{-1}=\int_{0}^{\eta} \int_{0}^{\eta} \int_{0}^{\eta} \int_{0}^{\eta}(). d \eta d \eta d \eta d \eta$, and $L_{2}^{-1}=\int_{0}^{\eta} \int_{0}^{\eta}(). d \eta d \eta$, 
let $f(0)=A, f^{\prime}(0)=B, f^{\prime \prime}(0)=C, f^{\prime \prime \prime}(0)=D, \theta(0)=E, \theta^{\prime}(0)=F$ we get,

$$
\begin{gathered}
f(\eta)=A+\eta B+\frac{\eta^{2}}{2} C+\frac{\eta^{3}}{3 !} D+L_{1}^{-1}\left\{S\left(\eta f^{\prime \prime \prime}+3 f^{\prime \prime}-2 f f^{\prime \prime \prime}\right)+M^{2} f^{\prime \prime}\right\}, \\
\theta(\eta)=E+\eta F+L_{2}^{-1}\left\{-\operatorname{Pr} S\left(2 f \theta^{\prime}-\eta \theta^{\prime}\right)-\operatorname{PrEc}\left(f^{\prime \prime 2}+12 \delta^{2} f^{\prime 2}\right)\right\},
\end{gathered}
$$

According to ADM, we have recurrence relation as

$$
\begin{array}{cc}
f_{0}(\eta)=A+\eta B+\frac{\eta^{2}}{2} C+\frac{\eta^{3}}{3 !} D, & \\
f_{k+1}(\eta)=L_{1}^{-1}\left\{S\left(\eta f_{k}^{\prime \prime \prime}+3 f_{k}^{\prime \prime}-2 A_{k}\right)+M^{2} f_{k}^{\prime \prime}\right\}, & k \geq 0, \\
\theta_{0}(\eta)=E+\eta F, & \\
\theta_{k+1}(\eta)=L_{2}^{-1}\left\{-\operatorname{Pr} S\left(2 B_{k}-\eta \theta_{k}^{\prime}\right)-\operatorname{PrEc}\left(C_{k}+12 \delta^{2} D_{k}\right)\right\}, & k \geq 0,
\end{array}
$$

where $A_{k}, B_{k}, C_{k}$ and $D_{k}$ are Adomian's polynomials

$$
\begin{gathered}
f_{1}(\eta)=L_{1}^{-1}\left\{S\left(\eta f_{0}^{\prime \prime \prime}+3 f_{0}^{\prime \prime}-2 A_{0}\right)+M^{2} f_{0}^{\prime \prime}\right\}, \\
\theta_{1}(\eta)=L_{2}^{-1}\left\{-\operatorname{Pr} S\left(2 B_{0}-\eta \theta_{0}^{\prime}\right)-\operatorname{PrEc}\left(C_{0}+12 \delta^{2} D_{0}\right)\right\} \\
f_{2}(\eta)=L_{1}^{-1}\left\{S\left(\eta f_{1}^{\prime \prime \prime}+3 f_{1}^{\prime \prime}-2 A_{1}\right)+M^{2} f_{1}^{\prime \prime}\right\}, \\
\theta_{2}(\eta)=L_{2}^{-1}\left\{-\operatorname{Pr} S\left(2 B_{1}-\eta \theta_{1}^{\prime}\right)-\operatorname{PrEc}\left(C_{1}+12 \delta^{2} D_{1}\right)\right\} \\
f_{3}(\eta)=L_{1}^{-1}\left\{S\left(\eta f_{2}^{\prime \prime \prime}+3 f_{2}^{\prime \prime}-2 A_{2}\right)+M^{2} f_{2}^{\prime \prime}\right\}, \\
\theta_{3}(\eta)=L_{2}^{-1}\left\{-\operatorname{Pr} S\left(2 B_{2}-\eta \theta_{2}^{\prime}\right)-\operatorname{Pr} E c\left(C_{2}+12 \delta^{2} D_{2}\right)\right\}
\end{gathered}
$$

where $A_{0}=f_{0} f_{0}^{\prime \prime \prime}$

$$
\begin{gathered}
A_{2}=f_{1} f_{0}^{\prime \prime \prime}+f_{0} f_{1}^{\prime \prime \prime},, \text { and }, \\
A_{1}=f_{2} f_{0}^{\prime \prime \prime}+f_{1} f_{1}^{\prime \prime \prime}+f_{0} f_{2}^{\prime \prime \prime}, \\
\cdot \\
\cdot \\
\cdot \\
B_{0}=f_{0} \theta_{0}^{\prime}, \\
B_{1}=f_{1} \theta_{0}^{\prime}+f_{0} \theta_{1}^{\prime} \\
B_{2}=f_{2} \theta_{0}^{\prime}+f_{1} \theta_{1}^{\prime}+f_{0} \theta_{2}^{\prime}, \\
\cdot \\
\cdot \\
\cdot \cdot \\
C_{0}=\left(f_{0}^{\prime \prime}\right)^{2}, \\
C_{1}=2 f_{0}^{\prime \prime} f_{1}^{\prime \prime}, \\
C_{2}=\left(f_{1}^{\prime \prime}\right)^{2}+2 f_{0}^{\prime \prime} f_{2}^{\prime \prime}, \\
\cdot \\
\cdot \\
\cdot \cdot \\
D_{0}=\left(f_{0}^{\prime}\right)^{2}, \\
D_{1}=2 f_{0}^{\prime} f_{1}^{\prime}, \\
D_{2}=\left(f_{1}^{\prime}\right)^{2}+2 f_{0}^{\prime} f_{2}^{\prime},
\end{gathered}
$$


The series solution is obtained as,

$$
\begin{gathered}
f(\eta)=f_{0}(\eta)+f_{1}(\eta)+f_{2}(\eta)+f_{3}(\eta) \ldots \\
f(\eta)=A+\eta B+\frac{\eta^{2}}{2} C+\frac{\eta^{3}}{3 !} D-\frac{1}{2520} S D^{2} \eta^{7}-\frac{1}{360} S C D \eta^{6}+\frac{1}{30} S D \eta^{5}+\cdots \\
\theta(\eta)=\theta_{0}(\eta)+\theta_{1}(\eta)+\theta_{2}(\eta)+\theta_{3}(\eta)+\cdots \\
\theta(\eta)=E+\eta F-\frac{1}{10} \delta^{2} \operatorname{PrEc} D^{2} \eta^{6}-\frac{1}{60} \eta^{5} \operatorname{PrSDF}-\frac{3}{5} \eta^{5} \operatorname{PrEcCD} \delta^{2}+\cdots
\end{gathered}
$$

\section{Results and Discussion}

Graph of $\theta(\eta)$ for $M=1.0=S=E c, \beta=\gamma=\delta=0.1$

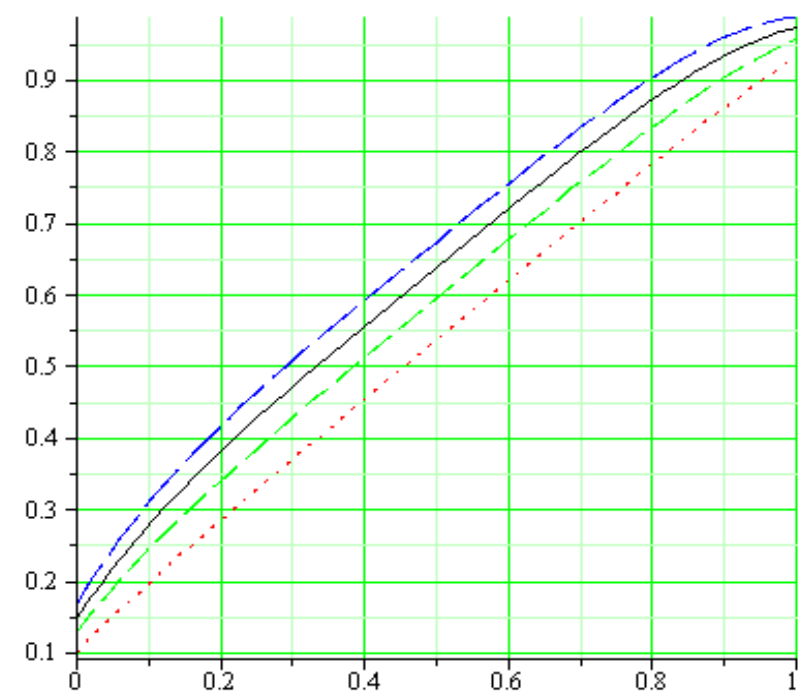

$\cdots \cdot \operatorname{Pr}=0.3--\operatorname{Pr}=0.8-\operatorname{Pr}=1.2--\operatorname{Pr}=1.5$

Figure 1. Effect of Prandtl number Pr on temperature profile

Graph of $f^{\prime}(\eta)$ for $\operatorname{Pr}=1.0=S, E c=0.5, \beta=\gamma=\delta=0.1$

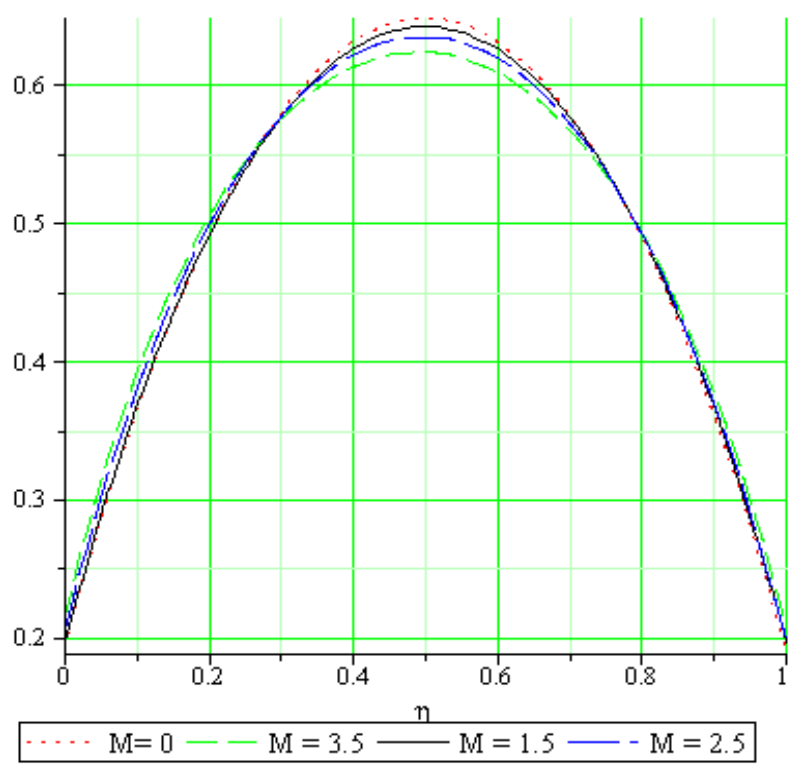

Figure 2. Effect of magnetic parameter $\mathrm{M}$ on the radial velocity 
Graph of $\theta(\eta)$ for $S=1.0=M=\operatorname{Pr}, E c=0, \beta=\delta=0.1$

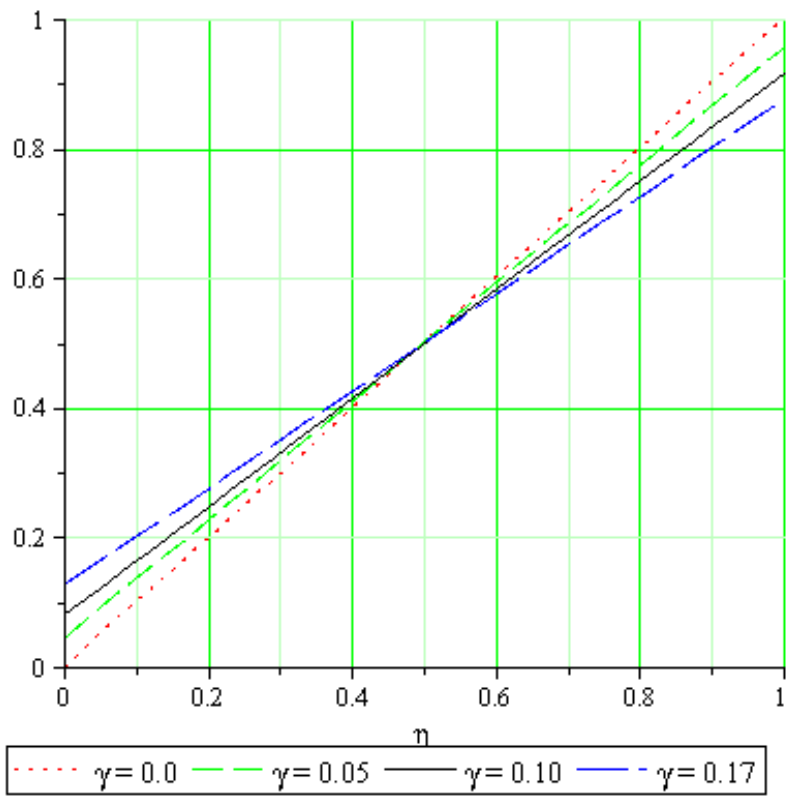

Figure 3. Effect of temperature slip parameter $\gamma$ on temperature profile

Graph of $\theta(\eta)$ for $S=1.0=M=\operatorname{Pr}, \beta=\delta=\gamma=0.1$

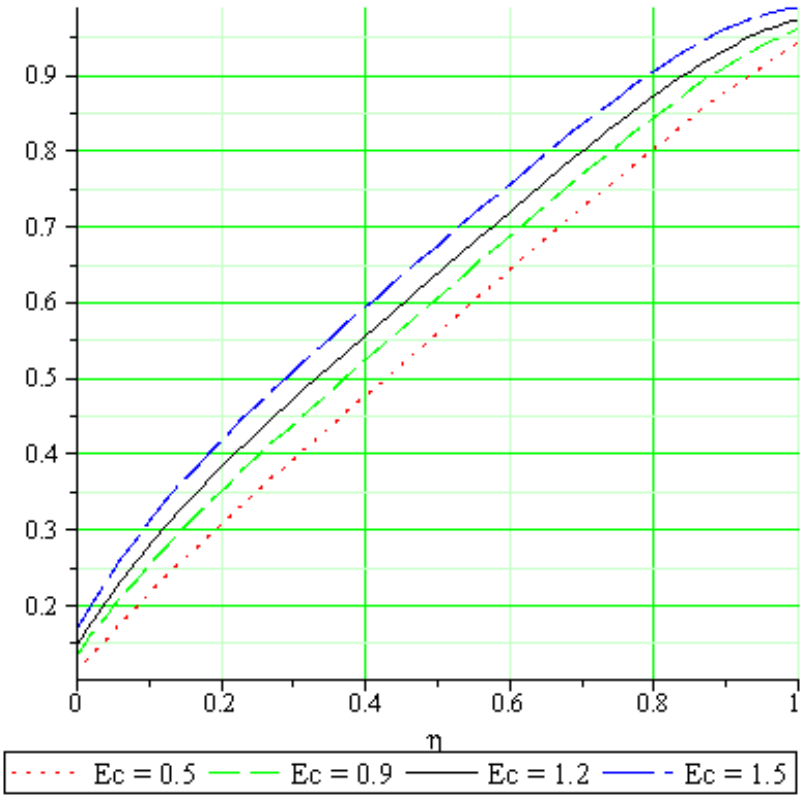

Figure 4. Effect of Eckert number Ec on temperature Profile

Graph of $f^{\prime}(\eta)$ for $\operatorname{Pr}=1.0=M, E c=0, \beta=\gamma=\delta=0.1$ 


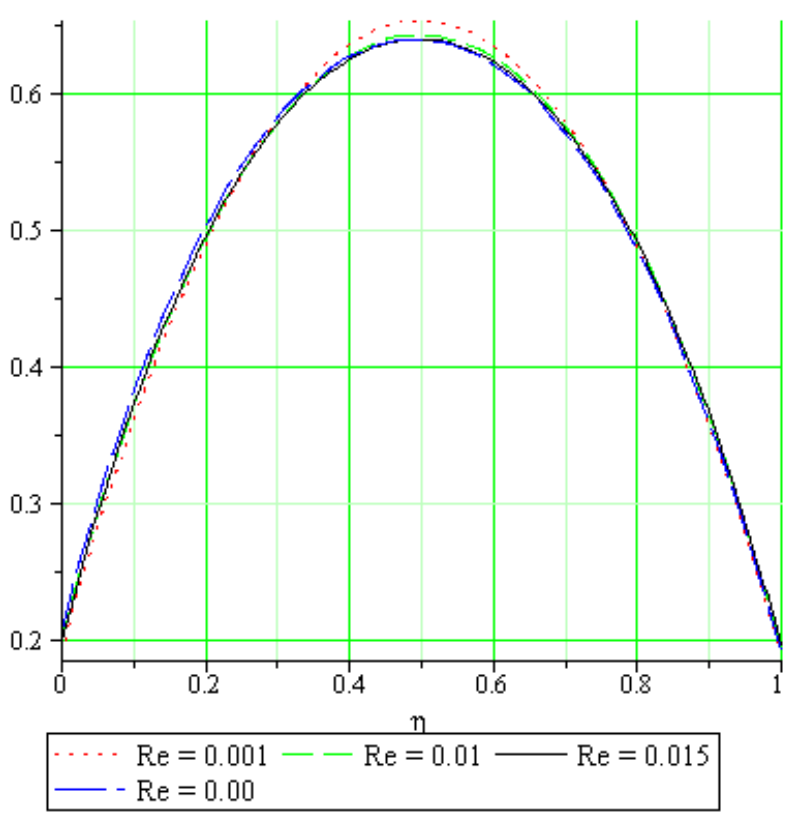

Figure 5. Effect of squeeze parameter on the radial velocity

Table 1 (a). Comparison of Numerical and different order ADM solutions for $f(\eta)$ when $S=M=\operatorname{Pr}=E c=1.0, \beta=\gamma=\beta=0.1$.

\begin{tabular}{ccccccccc}
\hline$\eta$ & $0^{\text {th }}$ order & $1^{\text {st }}$ order & $2^{\text {nd }}$ order & $3^{\text {rd }}$ order & $4^{\text {th }}$ order & $5^{\text {th }}$ order & $6^{\text {th }}$ order & $(\mathrm{NM})$ \\
0.0 & 0.00000 & 0.00000 & 0.00000 & 0.00000 & 0.00000 & 0.00000 & 0.00000 & 0.00000 \\
0.1 & 0.02679 & 0.02682 & 0.02839 & 0.02839 & 0.02838 & 0.02838 & 0.02837 & 0.02837 \\
0.2 & 0.06745 & 0.06788 & 0.07157 & 0.07157 & 0.07156 & 0.07154 & 0.07153 & 0.07153 \\
0.3 & 0.11748 & 0.11955 & 0.12537 & 0.12537 & 0.12536 & 0.12532 & 0.12532 & 0.12532 \\
0.4 & 0.17238 & 0.17852 & 0.18604 & 0.18604 & 0.18603 & 0.18598 & 0.18597 & 0.18597 \\
0.5 & 0.22767 & 0.24167 & 0.25007 & 0.25008 & 0.25007 & 0.25001 & 0.25000 & 0.25000 \\
0.6 & 0.27885 & 0.30587 & 0.31406 & 0.31411 & 0.31410 & 0.31403 & 0.31402 & 0.31402 \\
0.7 & 0.32143 & 0.36778 & 0.37460 & 0.37476 & 0.37475 & 0.37469 & 0.37468 & 0.37468 \\
0.8 & 0.35091 & 0.42372 & 0.42809 & 0.42853 & 0.42852 & 0.42847 & 0.42846 & 0.42846 \\
0.9 & 0.36279 & 0.46946 & 0.47059 & 0.47165 & 0.47165 & 0.47163 & 0.47163 & 0.47163 \\
1.0 & 0.35259 & 0.50000 & 0.49758 & 0.50000 & 0.50000 & 0.50000 & 0.50000 & 0.50000 \\
\hline
\end{tabular}

Table 1 (b). Comparison of Numerical and different order ADM solutions for $\theta(\eta)$ when $S=M=\operatorname{Pr}=E c=1.0, \beta=\gamma=\beta=0.1$.

\begin{tabular}{|c|c|c|c|c|c|c|c|c|}
\hline$\eta$ & $0^{\text {th }}$ order & $1^{\text {st }}$ order & nd order & $r d$ order 4 & order & $5^{\text {th }}$ order & $6^{\text {th }}$ order & (NM) \\
\hline 0.0 & 0.14709 & 0.14709 & 0.13489 & 0.13489 & 0.13809 & 0.13986 & 0.14012 & 0.14006 \\
\hline 0.1 & 0.29419 & 0.28001 & 0.25388 & 0.25388 & 0.26028 & 0.26382 & 0.26434 & 0.26423 \\
\hline 0.2 & 0.44128 & 0.39387 & 0.35096 & 0.35092 & 0.36054 & 0.36588 & 0.36666 & 0.36650 \\
\hline 0.3 & 0.58837 & 0.49929 & 0.43694 & 0.43664 & 0.44948 & 0.45663 & 0.45768 & 0.45747 \\
\hline 0.4 & 0.73546 & 0.60261 & 0.51880 & 0.51748 & 0.53351 & 0.54247 & 0.54379 & 0.54352 \\
\hline 0.5 & 0.88256 & 0.70602 & 0.60077 & 0.59701 & 0.61598 & 0.62675 & 0.62833 & 0.62801 \\
\hline 0.6 & 1.02965 & 0.80772 & 0.68458 & 0.67659 & 0.69784 & 0.71034 & 0.71219 & 0.71182 \\
\hline 0.7 & 1.17674 & 0.90206 & 0.76892 & 0.75587 & 0.77791 & 0.79191 & 0.79402 & 0.79360 \\
\hline 0.8 & 1.32383 & 0.97961 & 0.84796 & 0.83279 & 0.85301 & 0.86780 & 0.87013 & 0.86967 \\
\hline 0.9 & 1.47093 & 1.02731 & 0.90878 & 0.90294 & 0.91787 & 0.93160 & 0.93396 & 0.93351 \\
\hline 1.0 & 1.61802 & 1.02849 & 0.92733 & 0.95770 & 0.96496 & 0.97346 & 0.97506 & 0.97477 \\
\hline
\end{tabular}




\section{Conclusions}

Analytic and numerical techniques are used to analyze the influence of temperature and wall slip conditions on the unsteady MHD squeezing flow and heat transfer in a viscous fluid between two parallel disks. Analytical and numerical results are compared and an excellent agreement is found between them. In addition, Convergence of the series solution is demonstrated and it is found that fifth-order approximations are enough for a series solution. The results are plotted and following conclusions are drawn

- Increase in the value of velocity slip parameter $\beta$ results in increasing the radial velocity near the disks surfaces regions while opposite trend is observed near the central region.

- Skin friction coefficient is an increasing function of velocity slip parameter.

- Local Nusselt number increases with increasing value of temperature slip parameter.

\section{REFERENCES}

[1] M. J. Stefan, Versuch Uber die scheinbare adhesion, Akademie der Wissenschaften in Wien. Mathematisch-Naturwissenschaftliche, 69 (1874), 713.

[2] D. C. Kuzma, Fluid inertia effects in squeeze films, App. Sci. Res. 18 (1968), 15-20.

[3] E.A. Hamza, The magnetohydrodynamic squeeze film, J. Tribology, 110 (1988), 375-377.

[4] G. Domairry and A. Aziz, Approximate analysis of MHD Squeeze flow between two parallel disks with suction or injection by homotopy perturbation method, Math. Prob. Eng. (2009), DOI: $10.1155 / 2009 / 603916$.

[5] T. Hayat, A. Yousaf, M. Mustaf and S. Obaidat, MHD squeezing flow of second-grade fluid between two parallel disks, Int. J. Numer. Meth. Fluid, (2011), DOI: 10.1002/fld.2565.

[6] T. Hayat, M. Nawaz, A. A. Hendi and S. Asghar, MHD Squeezing Flow of a Micropolar Fluid Between Parallel Disks, J. Fluids Eng. 133 (2011), 111206.

[7] A. Qayyum, M. Awais, A. Alsaedi and T. Hayat, Unsteady squeezing flow of Jeffery fluid between two parallel disks, Chin. Phys. Lett. 29 (2012), 034701.

[8] A. R. A. Khaled and K. Vafai, Hydromagnetic squeezed flow and heat transfer over a sensor surface, Int. J. Eng. Sci. 42 (2004), 509-519.

[9] M. Mahmood, S. Asghar, M. A. Hossain, Squeezed flow and heat transfer over a porous surface for viscous fluid, Heat Mass Transf. 44 (2007), 165-173.

[10] H. M. Duwairi, B. Tashtoush, R. A. Damseh, On heat transfer effects in a viscous fluid squeezed and extruded between two parallel plates, Heat Mass Transf. 41(2004), 112-117.

[11] M. Mustafa, T. Hayat, S. Obaidat, On heat and mass transfer in the unsteady squeezing flow between parallel plates, Meccanica (2012), DOI: 10.1007/s11012-012-9536-3.

[12] B. Tashtoush, M. Tahat, S. D. Probert, Heat transfer and radial flows via a viscous fluid squeezed between two parallel disks, Appl. Energy, 68 (2001), 275-288.

[13] A. R. Bahadir, T. Abbasov, A numerical approach to hydromagnetic squeezed flow and heat transfer between two parallel disks, Ind. Lub. Trib., 63 (2011), 63-71.

[14] C. Neto, D. R. Evans, E. Bonaccurso, H.J. Butt, V.S.J. Craig, Boundary slip in Newtonian liquids: a review of experimental studies, Rep. Prog. Phys. 68 (2005), 2859-2897.

[15] C. L. M. H. Navier, The effect of slip condition on unsteady MHD oscillatory flow of a viscous fluid in a planer channel, Mem. Acad. Sci. Inst. France, 1 (1823), 414-416.

[16] C. S. Kumar, Microfluidic devices in nanotechnology: Fundamental concepts, John Wiley \& Son, New Jersey, 2009.

[17] A. Ebaid, Effects of magnetic field and wall slip conditions on the peristaltic transport of a Newtonian fluid in an asymmetric channel, Phys. Lett. A, 372 (2008), 4493-4499.

[18] C. Y. Wang, Analysis of viscous flow due to a stretching sheet with surface slip and suction, Nonlinear Anal. Real World Appl., 10 (2009), 375-380.

[19] K. Bhattacharyya, S. Mukhopadhyay, G. C. Layek, Slip effects on boundary layer stagnation-point flow and heat transfer towards a shrinking sheet, Int. J. Heat Mass Transf., 54 (2011), 308-313.

[20] M. H. Yazdi, S. Abdullah, I. Hashim, K. Sopian, Slip MHD liquid flow and heat transfer over non-linear permeable stretching surface with chemical reaction, Int. J. Heat Mass Transf., 54 (2011), 3214-3225.

[21] T. Hayat, M. Awais, A. A. Hendi, Three-dimensional rotating flow between two porous walls with slip and heat transfer, Int. Commun. Heat Mass Transf., 39 (2012), 551-555.

[22] L. Zheng, J. Niua, X. Zhang, Y. Gao, MHD flow and heat transfer over a porous shrinking surface with velocity slip and temperature jump, Math. Comput. Model., (2012), doi:10.1016/j.mcm.2011.11.080

[23] Y. Cherruault and G. Adomian, Decomposition methods: a new proof of convergence, Math. Comput. Model., 18(12) (1993), 103-106.

[24] A.M. Wazwaz, A reliable modification of Adomian's decomposition method, Appl. Math. Comput., 92(1) (1998), $1-7$.

[25] A. M. Wazwaz, Partial Differential Equations: Methods and Applications, Balkema Publishers, Leiden, (2002).

[26] A. M. Wazwaz, The modified decomposition method for analytic treatment of differential equations, Appl. Math. Comput., 173(1) (2006), 165-176.

[27] A. M. Wazwaz, A new algorithm for solving differential equations of the Lane-Emden type, Appl. Math. Comput., $118(2 / 3)(2001), 287-310$. 